\title{
A cathode-shared microbial fuel cell senor array for water alert system
}

Yong Jiang, Peng Liang*, Panpan Liu, Xiaoxu Yan, Yanhong Bian, and Xia Huang

State Key Joint Laboratory of Environment Simulation and Pollution Control School of

Environment, Tsinghua University, Beijing, 100084, P.R. China

*Corresponding author, Tel: +86 10 62796790; E-mail: liangpeng@tsinghua.edu.cn 


\begin{abstract}
:
The use of biosensors for water alert system is critical for providing safe water to the general public. A cathode-shared microbial fuel cell (MFC) sensor array was designed improve the detection credibility by eliminating the cathode performance variation, based on the principle that only the bioanode would be used as the sensing and transducing element. Four integrated MFCs exhibited high parallelism, and independence without the cross contamination or electric signal interference. Two linear ranges were observed (from 0.25 to $0.75 \mathrm{mM}$ and from 1 to $10 \mathrm{mM}$ ) for acetate detection. A linear relationship between inhibition ratio $(I R)$ and $\mathrm{Cu}^{2+}$ concentration from 2 to $6 \mathrm{mg} / \mathrm{L}$ was recorded. The sensor expressed an immediate voltage decrease when exposed to a $\mathrm{pH}$ decrease from 6 to 4 . The compact design of the cathode-shared MFC sensor array assure the detection credibility, and the number of the integrated MFC sensors is alterable based on the monitoring requirement.
\end{abstract}

\title{
Keywords:
}

Biosensor, microbial fuel cell, toxicity, detection credibility 


\section{Introduction}

On-line water alert system is critical for ensuring the proper operation of both drinking water and wastewater treatment plants, which is important to general public health. Conventional water quality monitoring using physicochemical analysis can precisely obtain the total elemental concentration of the target chemical compounds; however, it is impeded by the need for off-line analysis, long processing times, and lack of information on biological response. Various types of generic biosensors, which can reflect the actual bioavailability concentration of the target chemical compounds and the corresponding biological reactions, have been introduced into water alert system [1] Among these, the microbial fuel cell (MFC) based sensor has attracted growing interest because it can achieve self-sustainable and low-cost water monitoring without additional transducers, power sources or specialty chemicals [2-5]. The bioanode of an MFC is generally used as the sensing and transducing element, at which electrogenic microorganisms oxidize the organic substrate and release protons and electrons [6-9]. The electrons captured by the anode further transfer through the external circuit, whereas the protons diffuse through the solution to the cathode; here, electrons combine with protons and different electron accepters, e.g., oxygen and ferricyanide, to accomplish the reduction reaction [10-12]. The MFC sensors have been successfully used for organic matter and toxicity detection in water monitoring [2, 13]. However, from the standpoint of actual monitoring of water contamination events, the response time of MFC sensor is pretty long and detection credibility is difficult to guarantee, affected by the change of environmental parameters.

The reduction in response time can be generally achieved by optimizing the MFC sensor structure. For instance, by decreasing the volume of the anode chamber in an MFC from $25 \mathrm{ml}$ to $5 \mathrm{ml}$, leading to a lower hydraulic retention time (HRT), the 
response time can be significantly decreased from $36 \pm 2$ to $5 \pm 1$ min [14]. Accordingly, MFCs with a small anode chamber are expected to offer an inherent short response time [15]. However, there was few studies address the mathematical simulation of the flow pattern in the anode chamber of the MFC sensor, to illuminate the issue of how a decreased volume lead to a shorter response time. To increase the detection credibility, a more reliable analysis method is to increase the number of MFC sensors, probably using dozens of mini MFCs, to conduct parallel experiments and calibrate the output signal for other changing water quality parameters [16]. The MFC array with many MFCs integrated in a same platform for high-throughput testing, have been commonly used to select the electrogenic microorganisms [17]. Recently, some pioneer studies have reported the use of a compactly designed MFC array for water monitoring $[15,18$, 19].

The previously reported MFC array, however, suffered from non-parallelism, which decreased the detection credibility when used for water alert system. Although only the bioanode is used as the sensing and transducing element, the signal output of an MFC sensor is determined by the performance of both the anode and the cathode. In particular, the catalysis ability and stability of the cathode can affect the amplitude and stability of the background signal under non-toxic conditions, as well as the accuracy of the output signal under toxic conditions [20]. One of the common feature of the previously reported MFC array for water monitoring is that each of the MFC sensors has an independent anode as well as an independent cathode $[16,19]$, which potentially decreased their parallelism. One can expect that the detection credibility of a MFC array can be further improved by using cathode(s) with highly similar performance.

The objective of this study was to decrease the response time while increase the detection credibility of MFC sensors by providing a novel compactly designed 
cathode-shared MFC sensor array. The mathematical simulation of the flow pattern in the anode chamber was used here to illuminate the issue of how a decreased volume lead to a shorter response time. The multi-MFC sensors share the same large cathode to eliminate the cathode performance variation, taking advantage of the basic principle that only the bioanode is used as the sensing and transducing element in an MFC sensor for water monitoring $[4,21,22]$. With an emphasis on parallelism and independence, the performance of the MFC array was evaluated through the detection of organic matter, heavy metal $\left(\mathrm{Cu}^{2+}\right)$, and acidic toxicity. The compact design of the cathode-shared MFC sensor array was proved to have the promising of high-throughput testing with high detection credibility, by calibrating the effect of environmental changes during the field test.

\section{Materials and Methods}

\subsection{Construction of the cathode-shared MFC array}

The schematic of the cathode-shared MFC array with four anode chambers is shown in Fig. 1. A cation exchange membrane (CMI7000, Membranes International Inc., NJ, USA) was used to separate the cubic anode and cathode chambers. Each of the four anode chambers held a liquid volume of $1.4 \mathrm{~mL}$ and measured $1.5 \mathrm{~cm}$ in diameter by $0.8 \mathrm{~cm}$ in height, whereas the large shared cathode chamber held a liquid volume of $28 \mathrm{~mL}$ and measured $3 \mathrm{~cm}$ in diameter by $4 \mathrm{~cm}$ in height. The anodes were constructed of cylindrically shaped graphite felt (Sanye Carbon Co., Ltd., Beijing, China) and were $1.5 \mathrm{~cm}$ in diameter with a thickness of $0.2 \mathrm{~cm}$. The large cathode was a carbon-fiber brush $3 \mathrm{~cm}$ long and $3 \mathrm{~cm}$ in diameter. The wall of each anode chamber was drilled with two holes that served as the liquid inlet and outlet. The anolyte was forced to flow through the porous anodes as this proved to significantly improve the sensitivity of the MFC sensor [4]. Annular titanium sheets were used as the current collectors for these 
anodes and were held in place tightly by rubber gaskets. Both the anode and cathode chambers were replenishable to assure on-line and long-term analyses.

\section{Here Fig. 1}

\subsection{Startup of the cathode-shared MFC array}

The MFC array was inoculated with the effluent of an acetate-fed MFC in our laboratory $[23,24]$. The anolyte was prepared by dissolving $1.032 \mathrm{~g} \mathrm{Na}_{2} \mathrm{HPO}_{4} \cdot 12 \mathrm{H}_{2} \mathrm{O}$, $0.332 \mathrm{~g} \mathrm{NaH}_{2} \mathrm{PO}_{4} \cdot 2 \mathrm{H}_{2} \mathrm{O}, 0.13 \mathrm{~g} \mathrm{KCl}, 1.64 \mathrm{~g}$ sodium acetate, $0.125 \mathrm{~g} \mathrm{NH}_{4} \mathrm{Cl}, 12.5 \mathrm{~mL}$ trace minerals solution, and $5 \mathrm{~mL}$ vitamin solution in $1 \mathrm{~L}$ deionized water. The catholyte contained $100 \mathrm{mM} \mathrm{K}{ }_{3} \mathrm{Fe}(\mathrm{CN})_{6}$ in $50 \mathrm{mM}$ PBS solution in deionized water. Ferricyanide was used here rather than an air cathode because it is commonly used to provide readily available electron acceptors in MFC arrays [25]. Additionally, a previous study indicated that ferricyanide-based MFC array achieved higher parallelism compared to an air-cathode-based MFC array, probably due to the commonly observed performance degradation of air-cathode [26]. Both the anolyte and catholyte were recirculated at a constant flow rate of $3 \mathrm{~mL} \mathrm{~min}^{-1}$ using a multichannel peristaltic pump. The anodes were each connected to an external resistor with a fixed value resistance of $330 \Omega$ and were all further connected to the large shared cathode. According to our previous study [4], the MFC sensor could achieve higher sensitivity with a lower value of the external resistor. A preliminary experiment (data not shown) was conducted here, and it indicated that the cathode-shared MFC array could only achieve a stable operation when the value of the external resistor was higher than $330 \Omega$. Thus the commercial available fixed value resistance of $330 \Omega$ was used here.

\subsection{Biosensor test}

After enrichment of the anodic biofilms, the MFC sensors were then fed with a throughput of a sample stream during the process of the biosensor test. The anolyte was 
modified with specific concentrations of sodium acetate (as stated in Section 3) and fed into the anode chamber to test the MFC sensor in the area of organic matter detection. For heavy metal and acidic toxicity monitoring, the MFC array was challenged with anolyte containing specific concentrations of $\mathrm{Cu}(\mathrm{II})$ (note: copper chloride, $\mathrm{CuCl}_{2}$ ) and with acidified anolyte by hydrochloric acid to desired $\mathrm{pH}$ values, with the concentration of acetate consistently fixed at $5 \mathrm{mM}$. The using of copper here made it easy to compare our results with previous biosensor studies as it was widely used as the model heavy metal. All experiments were conducted in duplicate and performed at room temperature $\left(25 \pm 1^{\circ} \mathrm{C}\right)$.

\subsection{Analyses}

During the startup and biosensor tests, the voltage drop across the external resistor of $300 \Omega$ was measured every $5 \mathrm{sec}$ using a data acquisition system (DAQ2213, ADLINK, Beijing, China). Polarization curves and power density curves were obtained by varying the external resistance stepwise $(5,000,2000,1000,800,500,400,300,200$, and $100 \Omega$ ) at a time interval of $20 \mathrm{~min}$ [27]. The $\mathrm{pH}$ was monitored using a $\mathrm{pH}$ electrode (InLab® Micro, Mettler-Toledo, Switzerland). The flow pattern in the anode chamber of the MFC sensor was simulated using commercial ANSYS software (ANSYS, USA). The inhibition ration $(I R)$ is defined as the percentage of voltage drop normalized to the voltage before exposure to toxic agents and is calculated by the following equation [28]:

$$
\operatorname{IR}(\%)=100 \times\left(V_{\text {nor }}-V_{\text {tox }}\right) / V_{\text {nor }}
$$

where $V_{\text {nor }}(\mathrm{V})$ is the voltage generated before exposure to toxic agents and $V_{\text {tox }}(\mathrm{V})$ is the voltage output directly following the introduction of heavy metal $\left(\mathrm{Cu}^{2+}\right)$ and acidic toxicity.

\section{Results and Discussion}




\subsection{Current generation and power output}

To evaluate the performance of the MFC array, the current generation was recorded versus time as shown in Fig. 2a. During the three batch operations used to evaluate the cathode-shared MFC array, the relative standard deviations (RSDs) of the peak voltages were $4.5 \%, 4.9 \%$, and $5.3 \%$ at 4 hour, 28 hour and 63 hour, respectively, which were significantly lower than previous studies with RSDs from $14 \%$ to $22 \%$ [17, 26]. It was concluded that the current generation from each of the four MFCs integrated into an array was highly parallel. To further understand the power output capability, the polarization curves and power density curves were evaluated. As shown in Fig. 2b, a maximum power density of $1424 \pm 50 \mathrm{~mW} \mathrm{~m}^{-2}$ was obtained, which was 4 to 377 times higher than that of a previously reported MFC array $[17,25,29]$. The internal resistance was estimated at $1000 \Omega$ from the polarization curves, which was much lower than that of a previous MFC array used for water monitoring [16]. The high power output and low internal resistance could be attributed to the application of flow-through mode and the application of a large stable cathode, which were previously shown to increase the mass transfer inside the bioanode and avoid the limitations of cathode reactions, respectively [4]. More impressively, the four MFCs integrated into an array were highly parallel even when challenged with a large variation in external resistance, which could be attributed to the specific design of the cathode-shared structure that avoided the nonparallelism associated with separate cathodes.

\section{Here Fig. 2.}

\subsection{Detection of organic matter}

To evaluate the performance of the MFC array for organic matter monitoring, anolytes with increasing acetate concentration from 0 to $10 \mathrm{mM}$ were pumped into the four anode chambers integrated into an array (Fig. 3A). The detection limit, defined as 
the acetate concentration established using a 3:1 signal-to-noise ratio, was $0.25 \mathrm{mM}$ [30]. The response time, defined as the time required to reach $95 \%$ of the new steady-state signal output, varied from 5 to 25 minutes [14]. The flow pattern in the anode chamber after startup was simulated (Fig. S1) using the parameters obtained by switching the anolyte flow rate (Table. S1). The results indicated that decreasing the anode projected area and shrinking the anode chamber significantly stimulated mass transfer in the anode chamber. The response time increased to as long as $2 \mathrm{~h}$ merely by increasing the anode projected area and the anode chamber by 4-5 times (Fig. S2). A summary of double-chamber MFC sensors for organic matter detection in literature was shown in Table. 1. The response time observed here was much shorter than in previous studies as a result of the decreased anode volume and the application of flow-through mode $[3,31]$. However, the detection limit was at a moderate level, which might have been caused by the vitamin solution in the anolyte introducing a larger baseline signal [32]. Two linear ranges were observed: from 0.25 to $0.75 \mathrm{mM}$ and from 1 to $10 \mathrm{mM}$ (Fig. S3). The upper measurement limit obtained here was higher than that found in a previous study [33]. The phenomenon of two linear ranges was also observed in a recently study using microbial electrochemical technology for monitoring the volatile fatty acids [34]. The rate-limiting step of this two linear ranges was probably different. As the acetate increased, the rate-limiting step changed from the substrate concentration to the bioelectrochemical reaction ability, which was evidenced by a much lower slope of the linear fitting curve was obtained in the second ranges.

\section{Here Table. 1}

To evaluate the independence of the MFCs integrated into an array, the four anodes were further challenged with a different concentration of acetate between each MFC, as 
shown in Fig. 3B. The four MFCs exhibited high independence without the cross contamination from chamber to chamber or the electric signal interference potentially introduced by the shared cathode, all of which suggest the suitability of this configuration for use in higher-throughput tests.

\section{Here Fig. 3.}

\subsection{Detection of heavy metal $\left(\mathrm{Cu}^{2+}\right)$ toxicity}

To evaluate the performance of the MFC array for heavy metal toxicity monitoring, the four MFCs integrated into an array were challenged with different concentrations of $\mathrm{Cu}^{2+}$ at each MFC, as shown in Fig. 4. An immediate voltage decrease was recorded and a linear relationship between $I R$ and concentration was observed when the MFCs were exposed to $\mathrm{Cu}^{2+}$ in the range of 2 to $6 \mathrm{mg} / \mathrm{L}$. The detection limit observed here is in agreement with our previous study [4]. The MFC array was not reused to conduct the heavy metal toxicity test but run in duplicated reactors because it took at least $10 \mathrm{~h}$ to achieve full recovery depending on the concentration of $\mathrm{Cu}^{2+}$ (data not shown). The recovery performance observed here was also in agreement with previous studies [37, 38]. There are few reports in the literature that address an efficient recovery strategy. One practical way is to prepare parallel backup MFCs in case of irreversible inhibition or when a long recovery time is required.

\section{Here Fig. 4.}

\subsection{Detection of acidic toxicity}

After the MFC array reached steady state, an acidic toxicity shock was conducted using acidified anolyte. An immediate voltage decrease was observed when the $\mathrm{pH}$ decreased from 6 to 4, whereas the voltage of the control MFC was constant when the $\mathrm{pH}$ was kept at 7 . The performance of the MFC array exhibited a remarkably higher sensitivity compared with a previously reported MFC sensor, which only responded 
when the $\mathrm{pH}$ was as low as 4 [39]. The high sensitivity to acidic toxicity is probably caused by the decrease of anode volume from $28 \mathrm{~mL}$ to $1.4 \mathrm{~mL}$ and the application of flow-through mode. The relationship between the $I R$ and $\mathrm{pH}$ value was obtained for the first time in this study. However, it exhibited neither a linear relationship nor an exponential relationship, both of which are commonly observed in MFC sensors used in toxicity monitoring [15].

\section{Here Fig. 5.}

A cathode-shared MFC sensor array was designed here to improve the detection credibility by eliminating the cathode performance variation. Detection test of organic matter, heavy metal, and acidic toxicity indicated that the MFC sensors integrated in an array shown high parallelism and independence between each other. As a proof of concept, there were four MFC sensors integrated in the array. However, the number of the integrated MFC sensor is alterable based on the monitoring requirement of an alert system. For the practical usage of this novel cathode-shared MFC sensor array, one MFC sensor could be used as the reference sensor to calibrate the rest detection MFC sensors for environmental changes [16]. For instance, the environmental changes, e.g., temperature, during the field toxicity test could be calibrated by subtracting the response of the reference MFC sensor from that of the detection MFC sensors.

\section{Conclusions}

A cathode-shared MFC array was designed to eliminate the cathode performance variation, based on the principle that only the bioanode of an MFC sensor would be used as the sensing and transducing element. The four MFCs integrated in an array exhibited high parallelism as well as independence without the cross contamination or electric signal interference. Two linear ranges were observed (from 0.25 to $0.75 \mathrm{mM}$ and from 1 to $10 \mathrm{mM}$ ) for acetate monitoring with a response time that varied from 5 to 
25 min. A linear relationship between $I R$ and $\mathrm{Cu}^{2+}$ concentration from 2 to $6 \mathrm{mg} / \mathrm{L}$ was observed. The MFCs produced an immediate voltage decrease when the $\mathrm{pH}$ was decreased from 6 to 4 . Future research will focus on evaluating performance in more complex environments.

\section{Acknowledgments}

This work was supported by the National Science Foundation of China (NSFC No. $51278522,51422810)$.

\section{References}

[1] Su L, Jia W, Hou C, Lei Y. Microbial biosensors: a review. Biosens Bioelectron. 2011;26:1788-99.

[2] Kim M, Sik Hyun M, Gadd GM, Joo Kim H. A novel biomonitoring system using microbial fuel cells. J Environ Monit. 2007;9:1323-8.

[3] Kim BH, Chang IS, Gil GC, Park HS, Kim HJ. Novel BOD (biological oxygen demand) sensor using mediator-less microbial fuel cell. Biotechnol Lett. 2003;25:541-5.

[4] Jiang Y, Liang P, Zhang C, Bian Y, Yang X, Huang X, et al. Enhancing the response of microbial fuel cell based toxicity sensors to $\mathrm{Cu}$ (II) with the applying of flow-through electrodes and controlled anode potentials. Bioresour Technol. 2015;190:367-72.

[5] Jiang Y, Liang P, Liu P, Bian Y, Miao B, Sun X, et al. Enhancing Signal Output and Avoiding BOD/Toxicity Combined Shock Interference by Operating a Microbial Fuel Cell Sensor with an Optimized Background Concentration of Organic Matter. Int J Mol Sci. 2016;17:1392.

[6] Popov AL, Michie IS, Kim JR, Dinsdale RM, Guwy AJ, Esteves SR, et al. Enrichment strategy for enhanced bioelectrochemical hydrogen production and the prevention of methanogenesis. Int J Hydrogen Energy. 2016;41:4120-31.

[7] Sun D, Chen J, Huang H, Liu W, Ye Y, Cheng S. The effect of biofilm thickness on electrochemical activity of Geobacter sulfurreducens. Int J Hydrogen Energy. 2016;41:16523-8.

[8] Martinucci E, Pizza F, Perrino D, Colombo A, Trasatti SPM, Lazzarini Barnabei A, et al. Energy balance and microbial fuel cells experimentation at wastewater treatment plant Milano-Nosedo. Int J Hydrogen Energy. 2015;40:14683-9.

[9] Papaharalabos G, Greenman J, Melhuish C, Ieropoulos I. A novel small scale Microbial Fuel Cell design for increased electricity generation and waste water treatment. Int J Hydrogen Energy. 2015;40:4263-8.

[10] Logan BE, Hamelers B, Rozendal R, Schröder U, Keller J, Freguia S, et al. Microbial Fuel Cells: Methodology and Technology. Environ Sci Technol. 2006;40:5181-92.

[11] Jiang Y, Su M, Zhang Y, Zhan G, Tao Y, Li D. Bioelectrochemical systems for 
simultaneously production of methane and acetate from carbon dioxide at relatively high rate. Int J Hydrogen Energy. 2013;38:3497-502.

[12] Asghary M, Raoof JB, Rahimnejad M, Ojani R. A novel oxidizer-less and high performance microbial fuel cell by using DNA as a final electron acceptor in the cathodic chamber. Int J Hydrogen Energy. 2016;41:13611-8.

[13] Kim M, Youn SM, Shin SH, Jang JG, Han SH, Hyun MS, et al. Practical field application of a novel BOD monitoring system. J Environ Monit. 2003;5:640.

[14] Moon H, Chang IS, Kang KH, Jang JK, Kim BH. Improving the dynamic response of a mediator-less microbial fuel cell as a biochemical oxygen demand (BOD) sensor. Biotechnol Lett. 2004;26:1717-21.

[15] Sun JZ, Peter Kingori G, Si RW, Zhai DD, Liao ZH, Sun DZ, et al. Microbial fuel cell-based biosensors for environmental monitoring: a review. Water Sci Technol. 2015;71:801-9.

[16] Yang W, Wei X, Choi S. A two-channel bacteria-based biosensor for water quality monitoring. SENSORS, 2015 IEEE: IEEE; 2015. p. 1-4.

[17] Hou H, Li L, Cho Y, de Figueiredo P, Han A. Microfabricated microbial fuel cell arrays reveal electrochemically active microbes. PLoS ONE. 2009;4:e6570.

[18] Lee H, Yang W, Wei X, Fraiwan A, Choi S. A microsized microbial fuel cell based biosensor for fast and sensitive detection of toxic substances in water. Micro Electro Mechanical Systems (MEMS), 2015 28th IEEE International Conference on: IEEE; 2015. p. 573-6.

[19] Dávila D, Esquivel JP, Sabaté N, Mas J. Silicon-based microfabricated microbial fuel cell toxicity sensor. Biosens Bioelectron. 2011;26:2426-30.

[20] Stein NE, Hamelers HV, Buisman CN. Stabilizing the baseline current of a microbial fuel cell-based biosensor through overpotential control under non-toxic conditions. Bioelectrochemistry. 2010;78:87-91.

[21] Abrevaya XC, Sacco NJ, Bonetto MC, Hilding-Ohlsson A, Cortón E. Analytical applications of microbial fuel cells. Part I: Biochemical oxygen demand. Biosens Bioelectron. 2015;63:580-90.

[22] Abrevaya XC, Sacco NJ, Bonetto MC, Hilding-Ohlsson A, Cortón E. Analytical applications of microbial fuel cells. part II: Toxicity, microbial activity and quantification, single analyte detection and other uses. Biosens Bioelectron. 2015;63:591-601.

[23] Zhang C, Liang P, Yang X, Jiang Y, Bian Y, Chen C, et al. Binder-free graphene and manganese oxide coated carbon felt anode for high-performance microbial fuel cell. Biosens Bioelectron. 2016;81:32-8.

[24] Jiang Y, Liang P, Zhang C, Bian Y, Sun X, Zhang H, et al. Periodic polarity reversal for stabilizing the $\mathrm{pH}$ in two-chamber microbial electrolysis cells. Appl Energy. 2016;165:670-5.

[25] Hou H, Li L, Ceylan CU, Haynes A, Cope J, Wilkinson HH, et al. A microfluidic microbial fuel cell array that supports long-term multiplexed analyses of electricigens. Lab Chip. 2012;12:4151-9.

[26] Hou H, Li L, de Figueiredo P, Han A. Air-cathode microbial fuel cell array: a device for identifying and characterizing electrochemically active microbes. Biosens 
Bioelectron. 2011;26:2680-4.

[27] Jiang Y, Su M, Li D. Removal of Sulfide and Production of Methane from Carbon Dioxide in Microbial Fuel Cells-Microbial Electrolysis Cell (MFCs-MEC) Coupled System. Appl Biochem Biotechnol. 2014:1-12.

[28] Shen YJ, Wang M, Chang IS, Ng HY. Effect of shear rate on the response of microbial fuel cell toxicity sensor to $\mathrm{Cu}(\mathrm{II})$. Bioresour Technol. 2013;136:707-10.

[29] Choi S, Chae J. An array of microliter-sized microbial fuel cells generating $100 \mu \mathrm{W}$ of power. Sensors and Actuators A: Physical. 2012;177:10-5.

[30] Cheng L, Quek SB, Cord-Ruwisch R. Hexacyanoferrate-adapted biofilm enables the development of a microbial fuel cell biosensor to detect trace levels of assimilable organic carbon (AOC) in oxygenated seawater. Biotechnol Bioeng. 2014;111:2412-20. [31] Ayyaru S, Dharmalingam S. Enhanced response of microbial fuel cell using sulfonated poly ether ether ketone membrane as a biochemical oxygen demand sensor. Anal Chim Acta. 2014;818:15-22.

[32] Gai-Xiu Yang Y-MS, Xiao-Ying Kong, Feng Zhen, Ying Li, Lian-Hua Li, Ting-Zhou Lei, Zhen-Hong Yuan and Guan-Yi Chen. Factors affecting the performance of a single-chamber microbial fuel cell-type biological oxygen demand sensor. Water Sci Technol. 2013;68:1914-19.

[33] Kretzschmar J, Rosa LFM, Zosel J, Mertig M, Liebetrau J, Harnisch F. A microbial biosensor platform for in-line quantification of acetate in anaerobic digestion: potential and challenges. Chem Eng Technol. 2016;39:637-42

[34] Jin X, Angelidaki I, Zhang Y. Microbial electrochemical monitoring of volatile fatty acids during anaerobic digestion. Environ Sci Technol. 2016;50:4422-9.

[35] Chang IS, Jang JK, Gil GC, Kim M, Kim HJ, Cho BW, et al. Continuous determination of biochemical oxygen demand using microbial fuel cell type biosensor. Biosens Bioelectron. 2004;19:607-13.

[36] Quek S-B, Cheng L, Cord-Ruwisch R. Detection of low concentration of assimilable organic carbon in seawater prior to reverse osmosis membrane using microbial electrolysis cell biosensor. Desalin Water Treat. 2014:1-6.

[37] Stein NE, Hamelers HVM, Buisman CNJ. The effect of different control mechanisms on the sensitivity and recovery time of a microbial fuel cell based biosensor. Sensor Actuat B-Chem. 2012;171-172:816-21.

[38] Stein NE, Hamelers HVM, Buisman CNJ. Influence of membrane type, current and potential on the response to chemical toxicants of a microbial fuel cell based biosensor. Sensor Actuat B-Chem. 2012;163:1-7.

[39] Shen YJ, Lefebvre O, Tan Z, Ng HY. Microbial fuel-cell-based toxicity sensor for fast monitoring of acidic toxicity. Water Sci Technol. 2012;65:1223. 


\section{Figure captions}

Fig. 1. Schematic of the cathode-shared MFC array with four anode chambers. The number of the integrated MFC sensors is alterable based on the monitoring requirement.

Fig. 2. Current generation and power output of the MFC array. (A) Current generation versus time in three batch runs. (B) Polarization curves and power density curves of the cathode-shared MFC array.

Fig. 3. Performance of the MFC array for organic matter detection. (A) Voltage generation from an MFC array fed with anolyte containing different concentration of acetate. (B) Response of four MFCs integrated into an array challenged with different concentration of acetate between each MFC.

Fig. 4. (A) The MFC array used for $\mathrm{Cu}^{2+}$ toxicity monitoring. (B) Linear fitting results of inhibition ratio $(I R)$ to $\mathrm{Cu}^{2+}$ concentration.

Fig. 5. (A) The MFC array used for acidic toxicity monitoring. (B) Observed inhibition ratio $(I R)$ to $\mathrm{pH}$ values. 


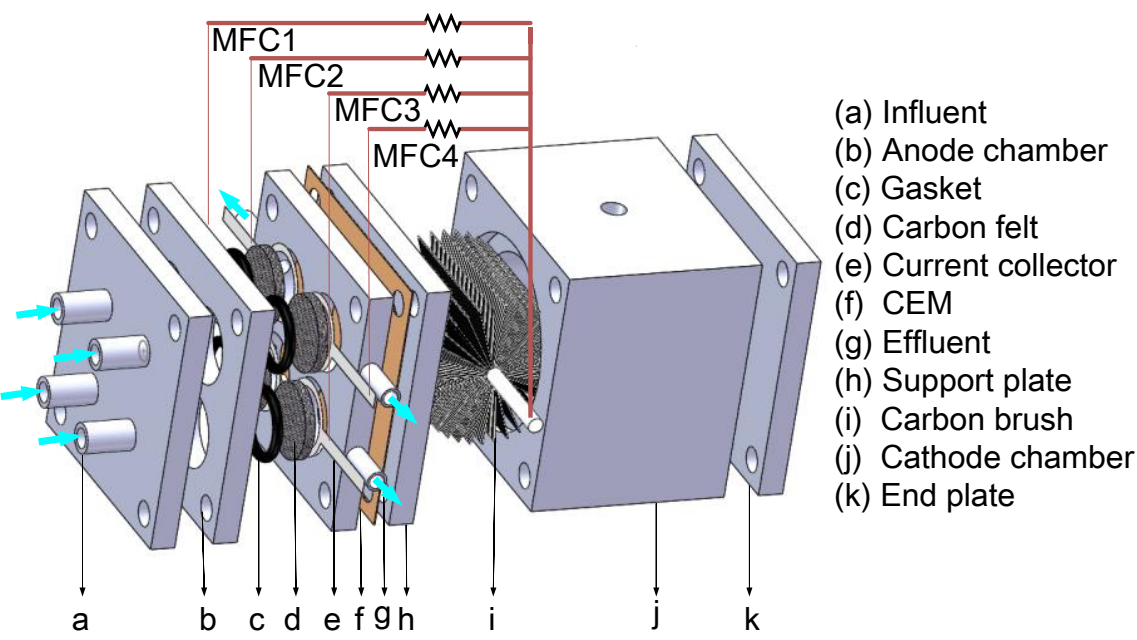

Fig. 1. 

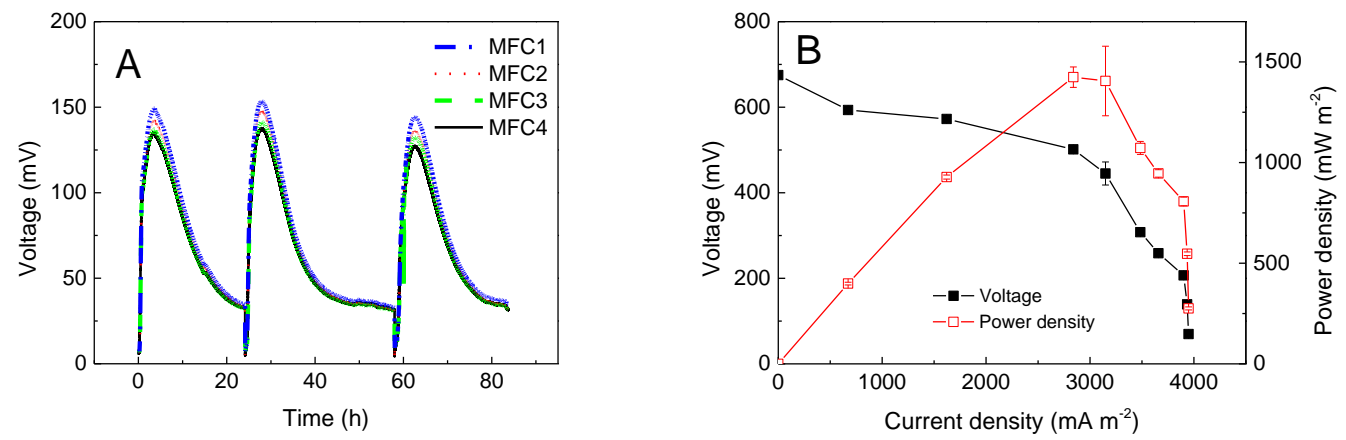

Fig. 2. 

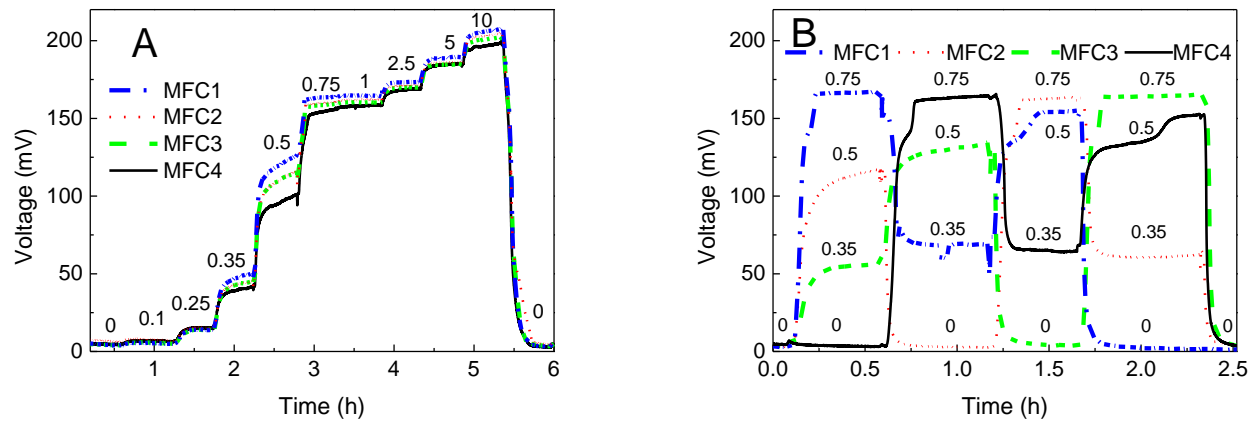

Fig. 3. 

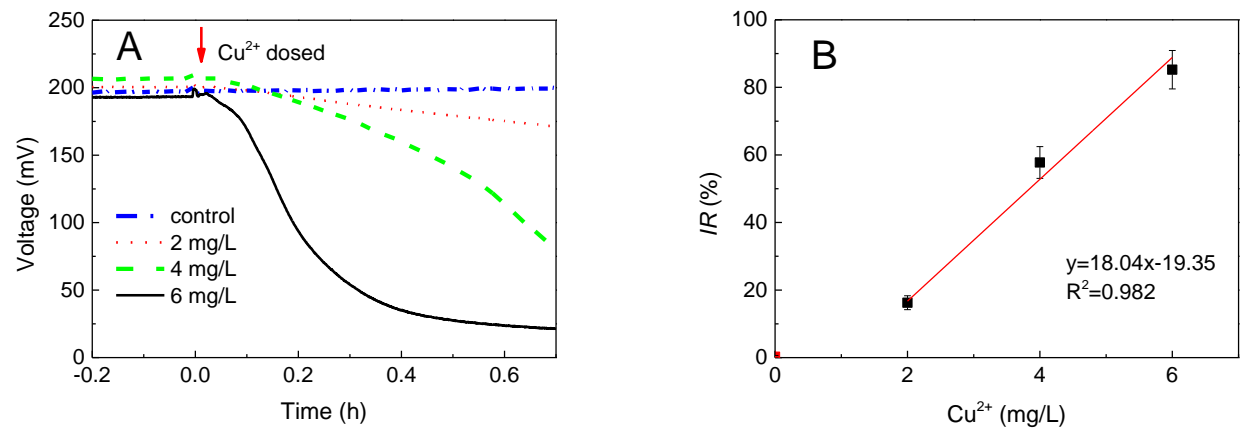

Fig. 4. 

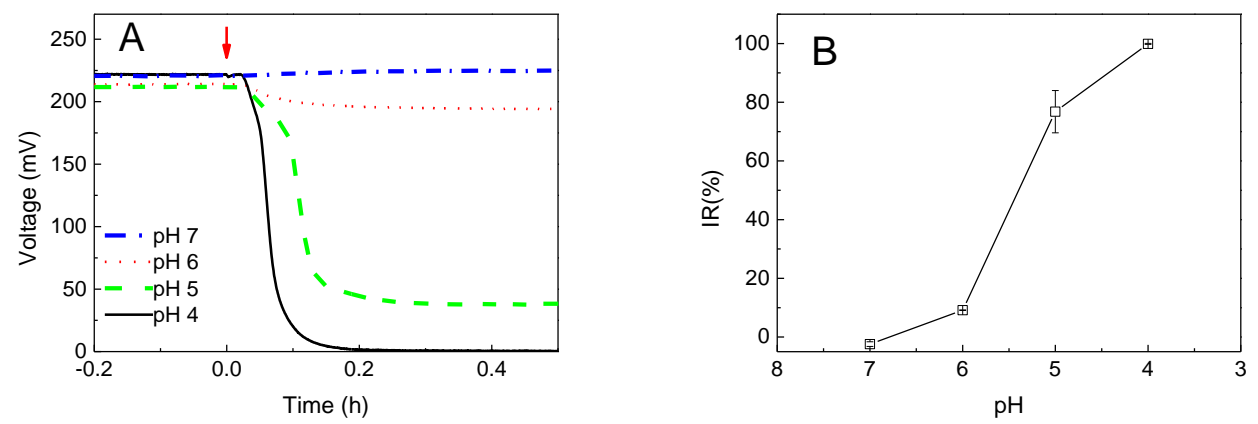

Fig. 5. 
Table. 1 Summary of double-chamber MFC sensors with similar dimension for organic

\begin{tabular}{ccccc}
\hline $\begin{array}{c}\text { Anode chamber } \\
(\mathrm{mL})\end{array}$ & inoculation & $\begin{array}{c}\text { linear range } \\
(\mathrm{BOD}, \mathrm{ppm})\end{array}$ & $\begin{array}{c}\text { Response } \\
\text { time }(\mathrm{min})\end{array}$ & Ref. \\
\hline 25 & MFC effluent & $20-200$ & 126 & {$[14]$} \\
25 & starch processing & $2.6-206$ & $30-600$ & {$[3]$} \\
20 & activated sludge & $20-100$ & 60 & {$[35]$} \\
54 & marine sediment & $0.64-10.88^{\mathrm{a}}$ & 60 & {$[36]$} \\
1.4 & MFC effluent & $16-640^{\mathrm{a}}$ & $5-25$ & This study \\
\hline
\end{tabular}

matter detection.

${ }^{\text {a }}$ theoretically calculated that $1 \mathrm{mM}$ acetate is equivalent to $64 \mathrm{ppm}$ BOD [24]; two linear ranges obtained here. 